\title{
Toward future photovoltaic-based agriculture in sea
}

\author{
Khaled Moustafa*
}

\begin{abstract}
To meet the challenges of climate change and water shortage, combining solar energy-based seawater desalination technologies with floating agriculture stations in one hybrid innovative system would be worthy of investigation for dry and sunny regions to desalinate seawater and produce valuable crops on board. Here, I discuss the feasibility of such a system as a 'floating farm' or 'bluehouse' in sea, by opposition to greenhouses in land. Potential advantages and shortcomings are debated.
\end{abstract}

Keywords: sea agriculture; sea farming; irrigation with seawater; global change; floating farm; floating field; seawater desalination; alternative agriculture; floating rice farms, climate change; environmental stress; drought; photovoltaic-based floating farm; bluehouses.

\section{Introduction}

Climate change and water shortage are important challenges for the future of humans and agriculture with substantial consequences on food security and environmental sustainability. Among the potential solutions to alleviate such challenges is to opt for vertical farming [1] and to breed new plant species potentially irrigable with seawater [2] [3] . Investors and researchers should also address any other potential approaches that would help developing sustainable farming systems to minimize the burden on freshwater and to increase plant productivity particularly in dry and arid lands where water cherishes.

With about $75 \%$ of the globe as a reserve, seawater is the most sustainable and inexhaustible source of water on Earth. However, in its natural state, saline water is unsuitable for productive agriculture and human consumption. To be usable, saltwater should be desalinated (purified from its excess salts) to produce freshwater suitable for irrigation and human uses. Solar energy-based seawater desalination is one of the most promising desalination approaches that could be used in sunny regions, either directly to produce water distillate in solar collectors, or indirectly by combining other conventional desalination techniques such as multistage flash desalination (MSF), vapor compression (VC), reverse osmosis (RO), membrane distillation (MD) or electrodialysis (ED) [4]. The availability of intensive solar energy in dry sunny regions over the year would make it possible to build 
large scale solar-based desalination facilities [ㄷ]. Although the quality of desalinated water produced from seawater might not be optimal for plant irrigation and human consumption, different treatments are available to stabilize it and to ensure its quality goals such recarbonation, disinfection and remineralization []].

\section{Photovoltaic floating farms as a potential agriculture/aquaculture solution}

In the context discussed above, a new hybrid system combining, in one structure, a solar energy-based seawater desalination approach with a 'floating farm' station would be worthy of investment for dry and sunny coastal regions to be implemented offshore as a 'floating marine field' or 'bluehouse' (by opposition to the greenhouse on land). A conceptual design of such a system is given in (Fig 1), and could be composed of three main parts or units; (1) sunlight capture unit (or photovoltaic) that captures sunlight and converts it into an electric power, (2) thermal desalination unit to desalinate seawater with energy produced in unit 1, and (3) a 'floating farm' unit with a cultivable surface (sandy, hydroponic or organic culture support) with walk-in growth rooms or corridors. The solar energy should first be collected by photovoltaic cells, and then converted into electric power that could be used to desalinate seawater via thermal approach (heating and condensation). The resulting freshwater will then be used to irrigate plants growing on board (Fig 1). Coupling other desalination approaches and/or alternative energy methods (i.e. wind turbine) to the photovoltaic-based desalination approach can also be combined to increase the overall amount of freshwater and energy produced.

To provide the most effective expertise toward building such systems, multidisciplinary investigations would be required, involving marine engineers, economists, mathematical modelers, system and mechanical engineers, biotechnologists, water purification and safety management specialists and plant biologist. In an ideal conception, photovoltaic-based floating farms could be designed to be immobile, semi-mobile, or completely mobile (automatically adjustable). However, mobile or adjustable systems will be costly but with major advantages over other options to respond to natural disasters, such as any decreases or increases in seawater levels or salt concentrations accumulated upon desalination process that should be diluted or discharged back into the seawater, far from the farming site.

Recently, prototypes of semi-transparent-bifacial photovoltaic modules for greenhouse roof applications were developed with a potential suitability for high-irradiation regions [7]. Moreover, the international BMT Design \& Technology group (Melbourne, Australia) is currently working on a floating desalination system with a potential scalability to produce up to 150 million liters per day []] Combing similar and movable desalination stations with floating farm surfaces onboard can thus be worthwhile investments for countries where freshwater is scarce and solar energy is plentiful over the year. 
Depending on the plant crops that will be cultivated in such systems (for e.g. cherry tomato, strawberry, spinach, lettuce, or even rice on appropriate support, etc.), precise calculations will be needed to set up optimal and proportional surfaces, volumes, farming supports, thickness and adequate physical and chemical characteristics of all the materials and components. For example, setting up a photovoltaic surface that should produce sufficient energy to fulfill all the energy requirements for $\mathrm{X}$ meter square/cube of culture surface over the whole life cycle of the growing plants, but also prior and posterior harvesting operations where energy and freshwater are also needed. Farming operations onboard and maintenance can also be fully automated. To do so, energy and freshwater should be produced in excess and stored to be used at time (beyond that, any excess of energy and freshwater can be transferred to neighboring land settings for human or industrial applications).

\section{Expected advantages}

Floating photovoltaic-based farms should offer many advantages over traditional soilbased farming systems, including for example the possibility to avoid high temperature and sandy storms' effects on desalination stations built on land in dry regions where such hurdles can harm the desalination system [9]. Since floating farms should be built with a full control of all the operations and growth conditions on board, compared with open soil-based farming where conditions are mostly out of control, floating fields should offer optimal conditions for growth and maturation, maximizing therefore the yield of growing crops. Moreover, and dislike open land farms that are exposed to all kinds of biotic and abiotic stressors, well-designed and isolated floating farms will reduce the risks of plant diseases and losses while avoiding the use of toxic pesticides. This can be achieved by using appropriate wall or barrier designs, in a similar way to hospital systems used to protect vulnerable patients, already suggested for use in an indoor farming [1]. To meet the quality criteria required for plant irrigation, the salts resulting from the desalination process can be reused in controlled and optimal proportions as mineral fertilizers for the floating farms after qualitative and quantitative analyses of the chemical composition of the recovered salts (remineralization of the desalinated water to ensure its quality). From an environmental perspective, floating farms in the sea will represent an important incentive for returning farmlands to their original ecological function (for e.g. reforestation) more than indoor or vertical farms intended to do so [1]]. Floating farms in the sea would also create unlimited farming surfaces (limited only by territory sea frontiers) and could reduce the burden on freshwater and the effects of climate change if adopted at large scales. To increase the profitability, high-valued crops or vegetables or rare medicinally efficient plants can eventually be tried for growing in such floating farms.

On the other hand, as fish aquaculture continues to grow and intensify worldwide, a photovoltaic-based aquaculture can also apply the potential approach outlined here. Solargenerated electric power can be used to meet the electric power requirements to automate 
all the operations of fish aquaculture systems such as for pumping water, aerating the cage and provide oxygen, purifying the water, and thus sustaining the aquaculture.

\section{Potential disadvantages}

The main drawback of floating photovoltaic-based farms is the cost, particularly at the first stages where multidisciplinary research and conceptions will be required to build solid and adapted structures that should resist hurricanes and seawater conditions. Owing to high costs of transferring maintenance stuffs and replacement materials to and from offshore sea-farm sites, floating farms should be designed to be more robustly adaptable to seawater conditions than land-based settings so they will require less maintenance operations but will increase the initial costs. An additional drawback is that, photovoltaicbased floating farms fits more with dry sunny regions than with cloudy, cold areas. However, in cold regions where arable surfaces are limiting factors, other desalination methods other than solar-energy such as filter-based pressure or reverse osmosis can be used to build 'floating bluehouses' and gain new farming surfaces in the sea.

Another potential pitfall is the low energy conversion rate that could be lower than optimal thresholds required for full control of an entirely automated floating farm system. However, to overcome this weakness, different sources of renewable energy can be combined to increase the overall energy generated, as it was recently experimented with photovoltaic-wind energy hybrid system to supply electric power [10]. Minimizing energy consumption [11] and increasing the conversion rates and conductivity of photovoltaic panels is indeed a matter of continual improvement. A table that summarizes the latest conversion rates of solar cells prototypes is published almost annually [12], illustrating the evolution of conversion rates that tend to be considerably enhanced year after year. The efficiency of the first generation of solar cells was less than 1\%, but since 1993 the conversion efficiency has been significantly improved for different types of solar cells, such as the silicon crystalline cells with up to $25.6 \%[\underline{13}]$, thin film with up to $28 \%$, and close to $40 \%$ for multi-junction devices [12]. In the next future, the conversion rate may be doubled or triplicated, making the conceptual system proposed here highly realizable and economically viable at least from a long-term perspective. Other problems in seawater desalination processes can be addressed in potential sustainable ways [14].

In summary, as the human activities and urbanization continue to grow, freshwater may become markedly scarce with harmful repercussions on agriculture. Researchers and stakeholders should explore innovative solutions and set up comprehensive plans to address the problem of agriculture sustainability and freshwater availability. Both sun and sea are inexhaustible sources for energy and water that could be approached smartly. Although 'floating farms' cannot replace farming on land at large scales for all types of agriculture, they can be considered as complementary solutions to the shrinkage of arable land and/or to produce some valuable vegetables or crops. Establishing floating farms in the sea would 
bring considerable environmental and sustainable advantages for next generations to face future climate challenges. This is particularly strategic for countries where freshwater and agriculture surfaces are dwindling because of crawling urbanism, weak rainfall rates and severe chronic drought. The advantages of such a system could far overweight its inconveniences at least from a long-term perspective.

\section{Disclosure: None}

\section{References}

1. Despommier, D., Farming up the city: the rise of urban vertical farms. Trends Biotechnol, 2013. 31(7): p. 388-9.

2. Moustafa, K., Toward breeding new land-sea plant hybrid species irrigable with seawater for dry regions. Plant Signal Behav, 2015. 10(4): p. e992744.

3. Moustafa, K., Binucleation to breed new plant species adaptable to their environments. Plant Signal Behav, 2015. 10(8): p. e1054586.

4. Qiblawey, H.M. and F. Banat, Solar thermal desalination technologies. Desalination, 2008. 220(1-3): p. 633-644.

5. Palenzuela, P., D.-C. Alarcón-Padilla, and G. Zaragoza, Large-scale solar desalination by combination with CSP: Techno-economic analysis of different options for the Mediterranean Sea and the Arabian Gulf. Desalination, 2015. 366(0): p. 130-138.

6. Withers, A., Options for recarbonation, remineralisation and disinfection for desalination plants. Desalination, 2005. 179(1-3): p. 11-24.

7. Yano, A., M. Onoe, and J. Nakata, Prototype semi-transparent photovoltaic modules for greenhouse roof applications. Biosystems Engineering, 2014. 122(0): p. 62-73.

8. Group BMT Design and Technology. 'Floating Desalination Plant'. Available from: http://www.bmtdesigntechnology.com.au/design-solutions/floating-desalination-plant/.

9. Reif, J.H. and W. Alhalabi, Solar-thermal powered desalination: Its significant challenges and potential. Renewable and Sustainable Energy Reviews, 2015. 48(0): p. 152-165.

10. Samrat, N.H., et al., Technical Study of a Standalone Photovoltaic-Wind Energy Based Hybrid Power Supply Systems for Island Electrification in Malaysia. PLoS One, 2015. 10(6): p. e0130678.

11. Subramani, A., et al., Energy minimization strategies and renewable energy utilization for desalination: a review. Water Res, 2011. 45(5): p. 1907-20.

12. Green, M.A., et al., Solar cell efficiency tables (version 46). Progress in Photovoltaics: Research and Applications, 2015. 23(7): p. 805-812.

13. Masuko, K., et al., Achievement of More Than 25\% Conversion Efficiency With Crystalline Silicon Heterojunction Solar Cell. Photovoltaics, IEEE Journal of, 2014. 4(6): p. 1433-1435.

14. Liyanaarachchi, S., et al., Problems in seawater industrial desalination processes and potential sustainable solutions: a review. Reviews in Environmental Science and Bio/Technology, 2014. 13(2): p. 203-214.

\footnotetext{
*Emai:khaled.moustafa@gmail.com
} 